\title{
Implementing Words Their Way with an Adolescent Who Uses AAC: A Case Study
}

\author{
Karissa Marble-Flint ${ }^{1}$, Holli Steiner ${ }^{2}$, Ashly Elliott ${ }^{2}$ Megan Stein $^{3}$ \\ Wichita State University ${ }^{1}$, Heartspring ${ }^{2}$, Salina Public Schools ${ }^{3}$ \\ United States of America
}

\begin{abstract}
Word study interventions (e.g., Words Their Way $\left.{ }^{\mathrm{TM}}\right)$ have been used for individuals with autism spectrum disorder (ASD) to support progress in reading. Although the literature strongly reinforces using word study interventions for reading decoding, their use for spelling has not been as widely researched. This case study project describes a student who communicated through augmentative and alternative communication (AAC) by typing novel utterances; however, his spelling did not follow conventional rules. Therefore, this created communication breakdowns for his communication partners since he could not utilize the spelling prediction features on his device. As a possible solution to this problem, the Words Their Way TM intervention was implemented to improve spelling. This student made progress in his spelling abilities, and more research is needed to generalize results to other AAC users.
\end{abstract}

\section{Introduction}

The speech-language pathologist (SLP) is one professional who is part of a multidisciplinary team providing intervention in the area of spelling. Spelling, a written language skill, is within the scope of practice of the SLP [1]. To support students who struggle to achieve spelling proficiency, one intervention approach that has been used by speechlanguage pathologists and educational professionals is word study. Word study has been defined as specifically concentrating on encoding (the letter and sound relationship) and decoding (sounding out words) for reading and spelling activities [2]. Word study intervention assists students' understanding of the relationship between sound structure and semantics [3]. Finally, it has been indicated that word study skills prompted better spelling abilities and Words Their Way ${ }^{\mathrm{TM}}$ (WTW) is one resource for implementation of word study [2]. What has not been widely studied is the use of word study interventions with individuals with ASD and users of AAC. This case study describes a word study intervention implemented by an SLP.

\section{Literature Review}

Research has indicated spelling proficiency requirements include phonemic and phonological awareness (understanding of sounds and parts of words separate from the meaning of the word), orthographic awareness (understanding of the letter sound relationship and which letters can be combined within words), morphological knowledge (understanding of base and root words and associated rules), and mental graphemic representations (a mental picture of those words that must be memorized as they do not follow phonological, orthographic or morphological rules) [4], [5], [6]. Smith Davis and Williamson [7] proposed a framework for instructional decisions which suggests that encoding (spelling and writing) and decoding (reading) should occur simultaneously. These tasks can assist the student in learning to read and increase accuracy in producing written words.

In addition, Smith Davis and Williamson [7] adapted spelling developmental stages for individuals with ASD from Bear et al. [8] and described the stages as phases. The term phases was used because there is not strong evidence to support the claim that individuals with ASD pass through the same spelling stages as typically developing peers [9].

Individuals who use AAC to communicate often experience difficulty acquiring written language skills, including spelling [10]. Word prediction on AAC devices has supported users' written language skills and has become commonplace on many personal devices such as smartphones. However, word prediction can, at times, slow down the rate of communication, create inaccuracies, and cause communication breakdowns if AAC users include too many spelling errors, particularly errors at the beginning of the word [11]. Therefore, specific focus on encoding and decoding skills can be valuable in this population, and one resource for such focus is WTW. 
A few researchers have explored the use of the WTW intervention for students with ASD. For example, a multiple baseline study completed by Solís et al. [12] included a modified WTW program for five participants with ASD in fifth through seventh grades. They found the WTW program to be effective in improving word reading skills. One researcher [13] implemented WTW for adolescents with ASD to determine its efficacy when used as a spelling intervention. The study included five males with a diagnosis of ASD between the ages of 15 and 19. Over six weeks, participants showed an average increase of $21 \%$ in spelling accuracy.

\section{Purpose}

The use of the WTW program for spelling intervention with individuals with ASD is an area of research that needs to be strengthened as there is a paucity of research surrounding spelling interventions implemented with individuals diagnosed with ASD who use AAC systems. The present case study aimed to evaluate the use of the WTW program for an individual with ASD who uses an AAC device to communicate. In addition, the current study analyzed one student diagnosed with ASD over a longer intervention period than a previous study of WTW conducted with adolescents with ASD [13].

The current case study adds to the literature by describing the implementation of a word study program for spelling, WTW, with a 17-year-old male who uses an AAC system. The aims of the study included the following: 1) to discuss the acquisition of spelling in typically developing peers and individuals with ASD, 2) to describe benefits of using word study intervention and WTW, and 3) to analyze the impact of WTW with an individual diagnosed with ASD who uses AAC.

\section{Method}

In this section, background information about the case study participant, his AAC evaluation, and a history of his previous AAC systems are provided in section 3.1. Next, an explanation of the procedures for the current study is detailed in section 3.2.

\subsection{Participant}

The current case study focused on a 17-year-old male with a diagnosis of ASD, intellectual disability, and a mild bilateral hearing impairment. At the time of the study, he lived and attended school at a residential and educational facility for students with special needs; he enrolled at the school in September of 2014. He appeared to enjoy socializing and sought out communication opportunities. He was non-verbal and used AAC to communicate with others.
His speech-language pathologist completed a formal AAC evaluation in November of 2014. According to the written report from that evaluation, he possessed skills which indicated he would be a candidate for a higher-tech communication system (i.e., he independently navigated his paper, no-tech communication book and required frequent additions to the book indicating that he would benefit from a speech generating device which would provide him with more efficient communication). At the time of the evaluation, he used American Sign Language (ASL) as his main mode of communication. In addition, he wore a point-and-carry no-tech communication book that consisted of sixteen pages with fifteen 1" x 1" Boardmaker pictures. Using ASL, he was understood by teachers/staff members approximately $20 \%$ of the time and understood $100 \%$ of the time when he used his communication book. He would also use pen and paper to communicate (his occupational therapist reported that his spelling was illegible due to inaccurate letter formation and letter spacing), but the report stated that communication breakdowns would often occur because of his lack of spelling skills. Based on the assessment, his SLP recommended the iPad with the TouchChat application (with the Word Power 80-page set) as a communication system. He received the system and communication application in 2015; the page set used with the application had access to a keyboard on most pages permitting him to use spelling as a communicative method when needed.

In 2015, he used the iPad system to answer a variety of questions, request, label, and communicate his wants and needs. During this time, it was observed that he would display behaviors surrounding the device. Examples included throwing the device when it was not charged and allowing the device to lose charge in order to get out of guided access (an iPad feature which allows the iPad to have an app locked on the screen). Per the residential and educational facility's policies, communication iPads were to be used only for communication and use guided access in order to allow the student use of only the communication application. It was hypothesized that he obsessed over the technology for multiple reasons which caused maladaptive behaviors.

Therefore, his special education team discussed several alternative communication options including the use of a full-time sign language interpreter, other high-tech AAC systems, and the use of a no-tech alphabet board. The team trialed a no-tech alphabet board with common phrases and then moved to the Tobii Dynavox Lightwriter SL40 Connect (see Figure 1), which is an AAC system with keyboard capabilities, in order to provide him with a more effective and efficient means to communicate as compared to a no-tech board. Because he previously had issues with tablet-based communication systems, 
his SLP made the decision to trial a system that was not tablet-based.

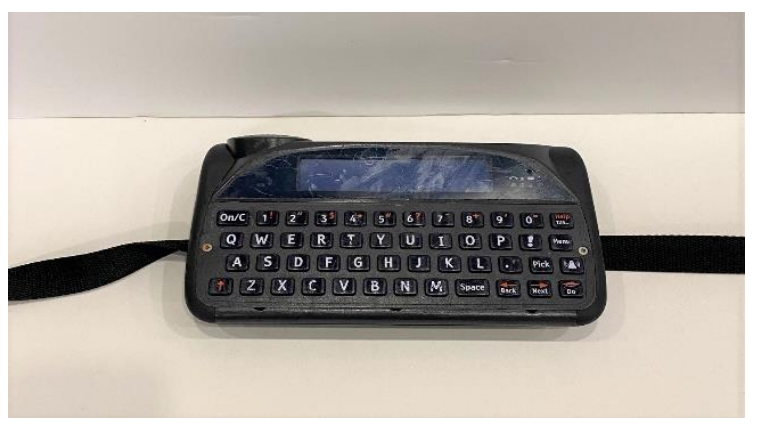

Figure 1. Tobii dynavox lightwriter

The Tobii Dynavox system included word prediction and a tactile communication board. His SLP suggested using a communication system with spelling capabilities because he had shown early spelling skills. In addition, the Tobii Dynavox system would allow him to create novel messages and not rely solely on chosen pictures from a system. His spelling often did not follow conventional rules which made it difficult for the device to use its built-in spelling prediction capabilities; this failure often led to communication breakdowns with his communication partners.

In 2019, since spelling had never been specifically targeted either educationally or through therapy services, his SLP looked for programs to trial in order to improve his spelling and therefore improve his ability to use his device and communicate with others. Therefore, to support spelling skills, the current study using WTW was initiated.

Following Institutional Review Board approval, the participant completed an assent form and his guardian signed a consent form. At the educational facility, the WTW intervention was implemented by the third author in a room separate from the participant's classroom. This was done to provide a quiet environment because the participant has hearing loss.

\subsection{Procedures}

The Test of Written Spelling, Fifth Edition (TWS5), Form A, was administered prior to the intervention. The TWS-5 is a norm-referenced assessment with two forms which can be used to show students' progress following an intervention. The authors used the assessment for this purpose. In addition, both the Primary and Elementary WTW Inventories were administered to determine the participant's spelling error patterns. The WTW Feature Guide was used to select a starting point for the intervention program and to establish the participant's developmental spelling age.
After administering the above assessments, intervention commenced. Across six months, the participant engaged with the third author, his speechlanguage pathologist (SLP), or a trained speechlanguage pathology assistant, in the WTW intervention for 20-minute sessions, five days a week.

Each week a different spelling pattern was introduced. With each spelling pattern (e.g., -ck, -ke, $-\mathrm{k})$, the third author used words from the WTW word list and administered a spelling test with 10 randomly selected words. Next, the participant was provided with the spelling list (15-20 words) for that week and each word was read aloud. The participant then sorted words (listed on notecards) according to spelling pattern after the SLP provided a model (see an example in Figure 2 below). The SLP checked the participant's work and provided corrections.

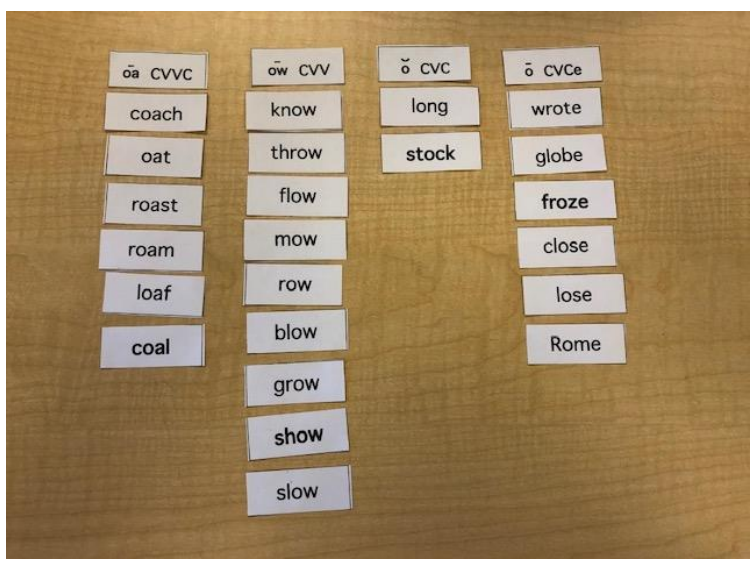

Figure 2. Example of word sort

After completing the word sort, the participant read a short story and circled words from the story that followed the spelling pattern. These procedures were repeated each week with a new spelling pattern. At the conclusion of each week, the SLP gave a spelling post-test of 10 randomly selected words from WTW. At the end of the six-month intervention period, Form B of the TWS-5 and the WTW Primary Spelling and Elementary Spelling Inventory post-tests were administered.

\section{Results}

The participant's performance on the standardized assessment, the TWS-5 Form A (administered prior to the intervention) and Form B (administered at the conclusion of the intervention) was compared. Results from the TWS-5 indicated that the participant's standard score increased by one. A Percentage of Words Correct (PWC) was calculated from the participant's performance on the Primary and Elementary Spelling Inventories, from WTW, which are curriculum-based measures. The participant's PWC increased by $8 \%$ on the Primary Spelling 
Inventory and by $4 \%$ on the Elementary Spelling Inventory.

Despite the fact that the standardized assessment did not yield significant change in spelling accuracy from pre-test to post-test, the post-test did reveal that the participant's misspellings were more closely representative of the target spellings than prior to intervention. Therefore, this led to additional analysis using a Spelling Sensitivity Score (SSS) [14]. These calculations were done using the SSS computerized program, which is available online [15].

The SSS computerized program produced both an Element (SSS-E) and Word (SSS-W) score. Elements consisted of the following three components: phonemes (speech sounds), juncture changes (spelling changes related to changes to a root word when adding a prefix or a suffix, e.g., the spelling change that happens to the word run when it changes to running), and affixes (i.e., prefixes and suffixes) [14]. See Table 1 for examples of words segmented by their elements. In multimorphemic words, as in the second and third words in Table 1, the root word is divided into phonemes, but the affix stays as one element. An element score was determined by the number of elements in the target word and the elements the participant produced. Each element was awarded points based on the rating system of 3 (correct), 2 (incorrect, but possible spelling), 1 (incorrect and not a possible spelling), and 0 (element is missing). After the points were calculated, the element points awarded were divided by the total number of elements possible to find the SSS-E score [14]. For this participant, his SSS-E pre-test average score was 2.38 and the post-test average score was 2.53 with an 0.15 increase in the overall average.

Table 1. Example Words Divided into Their Elements

\begin{tabular}{|c|c|}
\hline Example Word & Elements \\
\hline rain & r-ai-n \\
\hline running & r-u-n-n-ing \\
\hline jumped & j-u-m-p-ed \\
\hline
\end{tabular}

The SSS-W was determined by dividing the word points awarded by the total number of words in the sample. The SSS-W pre-test score was 1.62 and posttest was 1.88 . These scores indicated the participant's spellings were frequently phonologically correct; but additional work is needed on orthographic rules [14]. Overall, the increases in both the SSS-E and SSS-W were not significant; however, scoring using the SSS provided more detailed information on the participant's spelling patterns as compared to PWC. (see Table 2 for selected participant examples at pretest and post-test using SSS scoring.)

To gather qualitative results, the participant's classroom teacher shared information about his progress in an interview. His teacher reported that although the amount of spelling errors made by the participant had not changed, he did appear more willing to correct his errors than he did prior to the intervention. Initially, the participant accepted his spelling errors and "moved on" without correcting them. In addition, his teacher reported that following the intervention, he accessed his device to communicate more and wanted to discuss a more extensive range of topics. The teacher reported that participation in the WTW intervention seemed to have increased his confidence in his spelling abilities when communicating using his AAC systems.

Table 2. Words their way primary spelling inventory examples

\begin{tabular}{|c|c|c|c|c|c|}
\hline Targets & Pre-test & SSS-E & Post-test & SSS-E & $\begin{array}{l}\text { SSS- } \\
\text { E } \\
\text { Gain } \\
\text { Score }\end{array}$ \\
\hline rob & rib & 2.33 & rob & 3.00 & 0.67 \\
\hline coached & couth & 2.00 & $\cosh t$ & 1.67 & -0.33 \\
\hline chewed & caw & 1.00 & chew & 2.00 & 1.00 \\
\hline wishes & wishe & 2.50 & wished & 2.50 & 0.00 \\
\hline spoil & solid & 1.50 & spell & 2.25 & 0.75 \\
\hline camped & camups & 2.00 & camped & 3.00 & 1.00 \\
\hline tries & trys & 2.25 & trys & 2.25 & 0 \\
\hline clapping & calping & 2.17 & claping & 2.50 & 0.33 \\
\hline $\begin{array}{c}\text { SSS-E } \\
\text { Average } \\
\text { Score }\end{array}$ & 2.375 & & 2.525 & & 0.15 \\
\hline $\begin{array}{c}\text { SSS-W } \\
\text { Average } \\
\text { Score }\end{array}$ & 1.62 & & 1.88 & & 0.26 \\
\hline
\end{tabular}

\section{Discussion}

Overall, the participant's score demonstrated only a slight increase in PWC, yet assessing the participant's change in spelling accuracy using the SSS was crucial in analyzing how the participant's spelling accuracy had changed post-intervention. The PWC exclusively assessed correct or incorrect spelling. However, the SSS provided a more descriptive outline of the results and permitted a deeper understanding of the spelling errors. For example, when looking at the post-test Primary Spelling Inventory, the participant spelled the target 
word "coached" as "cosht." Using PWC, this spelling is incorrect. However, the SSS element score was 1.67 , giving more credit to the participant for his spelling of the word. As an example of a word in which the participant's score increased from pre-test to post-test and indicates his need to continue to work on orthography, the target word "clapping" was spelled as "calping" at pre-test and "claping" at posttest. Although this word was not accurate using PWC, using SSS element scoring, his score was 2.50. In addition, this spelling confirms that the participant was thinking in terms of phonemes (sounds) rather than graphemes (letters) It is possible that his spelling of this and other similar words may improve after direct instruction of spelling rules such as the "doubling rule". In summary, it is important to understand patterns of spelling errors and to not focus solely on the correct or incorrect spellings.

\section{Recommendations}

Recommendations for future studies include a longer duration for the intervention. It was found that WTW increased spelling accuracy for the participant slightly; however, a longer intervention duration with the word study may result in greater improvements. Other research questions to consider with the implementation of an increased study duration would include the following:

2. Would the participant continue making progress with more complex spelling patterns?

3. Would the participant's progress on previously mastered spelling patterns be sustained?

4. Would the participant's previously mastered spelling patterns generalize to other words with similar spelling patterns?

5. Would the participant continue to require frequent, intensive intervention sessions to continue making progress (i.e., if he attended sessions 3 times a week instead of 5 times a week)?

Further, the teacher indicated in a postintervention interview that she felt his confidence in his spelling abilities had improved, yet data was not collected in this area. With a longer intervention period, pre- and post-intervention data could be collected using a Likert-type scale about communication partners' (e.g., teacher, classroom paraeducator, parent, etc.) attitudes/views/feelings about his spelling abilities and spelling confidence to supply qualitative data to complement the quantitative data.

In addition, future studies with participants who demonstrate similar needs may replicate these procedures and include a focus on the alphabetic principle prior to implementing WTW. This study did not investigate the participant's prior knowledge about the alphabetic principle before implementing the WTW program.

In addition, this study was conducted with one participant, but it is possible that other implementers of this program would need to use it in small groups of adolescents who use AAC. Further data needs to be collected to determine if this program would be as effective for AAC users in small group learning settings versus individual student learning.

\section{Conclusion}

WTW may be beneficial for spelling skills with other adolescents who use AAC, but further research is needed. Speech-language pathologists and educators will need to assess the individual needs of students to determine if WTW should be implemented. Further, the link between spelling skills and users of AAC remains a research area in need of strengthening. To determine the educational implications of using WTW with individuals with autism who use AAC, additional research is needed.

\section{References}

[1] American Speech-Language-Hearing Association. (2016). Scope of practice in speech-language pathology [Scope of Practice]; https://www.asha.org/policy/sp201600343/\#Domains (Access Date: 14 January, 2021).

[2] Apel, K. "Word study and the speech-language pathologist." Perspectives on Language Learning and Education 11(3), 2004. pp. 13-17.

[3] Paul, R., and Norbury, C. Language disorders from infancy through adolescence: listening, speaking, reading, writing, and communicating (4th ed). Mosby, St. Louis, MO, 2012.

[4] Apel, K.; Masterson, J. "Theory-guided spelling assessment and intervention: A case study." Language, Speech, and Hearing Services in Schools, 32, 2001. pp.182195.

[5] Hart, P., Scherz, J., Apel, K., and Hodson, B. Analysis of spelling error patterns of individuals with complex communication needs and physical impairments, Augmentative and Alternative Communication 23(1), 2007. pp. 16-29.

[6] Shipley, K. G., and McAfee, J. G. Assessment in speechlanguage pathology: A resource manual. Cengage Learning, Boston, MA, 2016.

[7] Smith Davis, H. and Williamson, P. "Word recognition". In C. Carnahan and P. Williamson (Eds.), Quality literacy instruction for students with autism spectrum disorders (pp. 253-285). AAPC Textbooks, Shawnee Mission, KS, 2010.

[8] Bear, D. R., Invernizzi, M., Templeton, S., and Johnston, F. Words their way: Word study for phonics, vocabulary 
and spelling instruction (4th ed.). Pearson, Upper Saddle River, NJ, 2008.

[9] Gabig, C. S. "Phonological awareness and word recognition in reading in children with autism." Communication Disorders Quarterly, 31(2), 2010. pp. 6785.

[10] Hart, P., "Spelling Considerations for AAC Intervention." Perspectives on Augmentative and Alternative Communication. 15(2). 2006, pp. 12-14.

[11] Cler, G.J., Kolin, K.R., Nordzij, J. P., Jr., Vojtech, J.M., Fager, S.K., and Stepp, C.E. "Optimized and Predictive Phonemic Interfaces for Augmentative and Alternative Communication." Journal of Speech, Language, and Hearing Research. 62, 2019. pp. 2065-2081.

[12] Solís, M., El Zein, F., Black, M., Miller, A., Therrien, W. J., and Invernizzi, M. "Word Study Intervention for Students with ASD: A Multiple Baseline Study of DataBased Individualization." Education and Training in Autism and Developmental Disabilities, 53(3), 2018, pp. 287-298.

[13] Gutknecht, E. A. Exploring the Effects of Using the Words Their Way Program with Teenage Boys with Autism. (Master's Thesis), 2015. (Access Date: 3 November, 2020).

[14] Masterson, J. J., and Apel, K. "The Spelling Sensitivity Score: Noting Developmental Changes in Spelling Knowledge." Assessment for Effective Intervention, 36(1), 2010, pp. 35-45.

[15] Masterson, J. and Hrbec, B. Computerized Spelling Sensitivity [computer software]. Missouri State University Language-Literacy Lab; Springfield, MO: 2011.

\section{Acknowledgments}

We would like to thank the participant, his guardian, his teacher, and his paraeducator for their involvement in this project. 